Machine Learning and Deep Learning

Subscriber access provided by UNIVERSITAT POLITÈCNICA DE VALÈNCIA

\title{
Identification of New Templates for the Synthesis of BEA, BEC and ISV Zeolites Using Molecular Topology and Monte Carlo Techniques
}

Maria Galvez-Llompart, Jorge Galvez, Fernando Rey, and German Sastre

J. Chem. Inf. Model., Just Accepted Manuscript • DOI: 10.1021/acs.jcim.0c00231 • Publication Date (Web): 27 May 2020

Downloaded from pubs.acs.org on June 1, 2020

\section{Just Accepted}

"Just Accepted" manuscripts have been peer-reviewed and accepted for publication. They are posted online prior to technical editing, formatting for publication and author proofing. The American Chemical Society provides "Just Accepted" as a service to the research community to expedite the dissemination of scientific material as soon as possible after acceptance. "Just Accepted" manuscripts appear in full in PDF format accompanied by an HTML abstract. "Just Accepted" manuscripts have been fully peer reviewed, but should not be considered the official version of record. They are citable by the Digital Object Identifier (DOI®). "Just Accepted" is an optional service offered to authors. Therefore, the "Just Accepted" Web site may not include all articles that will be published in the journal. After a manuscript is technically edited and formatted, it will be removed from the "Just Accepted" Web site and published as an ASAP article. Note that technical editing may introduce minor changes to the manuscript text and/or graphics which could affect content, and all legal disclaimers and ethical guidelines that apply to the journal pertain. ACS cannot be held responsible for errors or consequences arising from the use of information contained in these "Just Accepted" manuscripts. 


\title{
Identification of New Templates for the Synthesis of BEA, BEC and ISV Zeolites Using Molecular Topology and Monte Carlo Techniques
}

\author{
María Gálvez-Llompart ${ }^{1,2}$, Jorge Gálvez ${ }^{2}$, Fernando Rey ${ }^{1}$, German Sastre $^{1 *}$
}

\footnotetext{
${ }^{1}$ Instituto de Tecnología Química (UPV-CSIC), Universidad Politécnica de Valencia; Av. Naranjos s/n, 46022 Valencia, Spain. e-mail: gsastre@itq.upv.es

${ }^{2}$ Molecular Topology and Drug Design Unit, Department of Physical Chemistry, University of Valencia, Valencia, Spain.
} 


\begin{abstract}
The presence of organic structure directing agents (templates) in the synthesis of zeolites allows the synthesis to be directed, in many cases, towards structures in which there is a large stabilisation between the template and the zeolite micropore due to dispersion interactions. Although other factors are also important (temperature, $\mathrm{pH}, \mathrm{Si} / \mathrm{Al}$ ratio, etc), systems with strong zeolite-template interactions are good candidates for an application of new computational algorithms, for instance those based in molecular topology (MT), that can be used in combination with large databases of organic molecules. Computational design of new templates allows the synthesis of existing and new zeolites to be expanded and refined. Three zeolites with similar 3D large pore system, BEA, BEC and ISV were selected with the aim of finding new templates for their selective synthesis. Using a training set of active and inactive templates (obtained from literature) for the synthesis of target zeolites it was possible to select chemical descriptors related to activity, meaning a good candidate template. With a discriminant function defined upon MT, the screening through a database of organic molecules led to a small subset (preselection) of candidate templates for the synthesis of BEA, BEC and ISV. As far as we know, this is the first time that topological/topochemical descriptors, which do not consider 3-D information of the molecules, have been used to predict the activity of zeolite structure directing agents (SDA). Following the prediction of SDAs using MT, an automated approach of sequential template filling of micropores based on a combination of Monte Carlo and lattice energy minimization was applied for all the candidate templates in the three zeolites. Two results can be obtained from this: an evaluation of the quality of the molecular topology QSAR models leading to the preselection of templates, and, a final selection of candidate templates for the selective synthesis of BEA, BEC and ISV. Regarding the latter, a good template will be that which maximizes the zeolite-template dispersion interactions with one, and only one, of the three zeolites. The presented methodology can be used to find alternative (maybe cheaper or perhaps more selective) templates than those already known.
\end{abstract}




\section{Introduction}

Zeolite materials are constituted by corner-sharing $\mathrm{TO}_{4}$ tetrahedra $(\mathrm{T}=\mathrm{Si}, \mathrm{P}, \mathrm{Al}, \mathrm{Ge}, \mathrm{Ga}$, etc) giving a regular micropore system. ${ }^{1}$ These crystalline inorganic microporous solids are widely used in catalysis, adsorption, separation, microelectronics, and ion-exchange. Moreover, zeolitic materials are used in fields such as luminescence, electricity, magnetism and medicine. This extensive realm of zeolite applications arises from their peculiar chemical compositions and unique porous channels, which are classified as small, medium, large, and extra-large, with respective pore windows being delimited by $8,10,12$, and more than $12 \mathrm{~T}$-atoms. ${ }^{2}$

Many variables influence the synthesis process of a zeolitic material, making it difficult to establish a relationship between synthetic variables and the particular zeolite structures formed. ${ }^{3}$ Although synthesis parameters, such as crystallization time, temperature and gel composition (Tatom source), structure-directing agents (SDA), mineralization agent, solvent, and concentration, are known to be relevant in the process, a rational selection of the parameters necessary to drive the synthesis towards a specific zeolite is yet not possible.

Among the molecules used as SDAs, those of organic origin (OSDA) stand out. Different properties such as shape, size, charge, rigidity, polarity and hydrophobicity of organic structuredirecting agents (OSDA), also called 'templates', can modify the resulting pore openings, pore dimensions and connectivities, as well as pore architectures of the synthesized zeolites. The idea of shape and size match between the template and the zeolite micropore allows a rational choice of organic SDAs that could lead, at least in some particular cases, to synthesis by design of zeolite structures. ${ }^{45}$

Although small simple molecules, such as tetraalkylammonium cations, were utilized as SDAs, most of them have complex structures containing units such as rigid rings and flexible chains and are prepared following laborious reaction schemes, making them not commercially available. ${ }^{6}$ Therefore, the use of commercially available SDAs or SDAs obtained through simple synthesis processes is increasingly demanded.

The design of appropriate templates for the synthesis of specific zeolites has been a holy grail of zeolite science since the late nineties. Before computational design was even suggested, a computational methodology had to be developed, with the groups of $\mathrm{Catlow}^{7}$, $\mathrm{Casci} / \mathrm{Cox}^{8}$, and Davis/Zones ${ }^{9}$ being pioneers, and with the latter in particular very active ever since ${ }^{10-13}$. Soon after, arguably the first computational method to design templates was proposed ${ }^{14}$, although applied few times. An early example is the design of templates for zeolite NU-87 (NES) ${ }^{15}$, and more recently for derivatives of AFX and related structures ${ }^{16}$. Deem has been working successfully on the topic since 2013, with examples such as designing templates for AEI ${ }^{17}$, $\mathrm{STW}^{18}$, and $\mathrm{BEA}^{19}$. The strategies developed are particularly consistent since they add the possibility to screen throughout a database of templates ${ }^{20}$, or to design libraries of templates from scratch $^{21}$. In our group, an early study was among the first to consider the presence of aluminum and its location on template stabilization ${ }^{22}$, we have carried out extensive work focused on 
particular structures such as EU-1 (EUO), ZSM-11 (MEL) and ZSM-12 (MTW) ${ }^{23,24}$. More recently we have included artificial intelligence and big data analysis in our own software ${ }^{25}$.

Computational chemistry has become an important tool in assisting the discovery of new OSDAs for zeolite synthesis. Some of the most common computer-aided techniques already applied to OSDA identification are Monte Carlo, energy minimization studies, molecular dynamics, molecular docking, de novo design and machine learning models. ${ }^{25-28}$ The recent work by Daeyaert et al. employs a machine learning technique to train a large set of SDAs that experimentally give Beta zeolite, in order to find a larger set of SDAs for the same zeolite. ${ }^{27}$ The training set relates the structure of the SDA with its stabilization energy in Beta, with no need to make the computationally expensive calculation of the zeo-SDA energy.

Computational techniques based on graph theory have recently been used to find similarities and invariants that allow common aspects between zeolite intergrowths to be found, and to group zeolites into families according to new topological definitions..$^{29,30}$

QSAR or QSPR studies(quantitative structure activity or property relationships, respectively) are among thecomputational techniques applied to this field of chemistry. These techniques are based on relating the chemical structure of compounds to a certain biological activity, or physicochemical or other experimental properties by means of descriptors. When these descriptors are topological, the approach is known as QSAR based on molecular topology (MTQSAR), which employs graph theory to characterize chemical compounds by means of topological or topochemical descriptors, that may include 2-D information of the molecule but not the 3-D coordinates. To generate the topological descriptors the starting point is describing the molecules by graphs, which in turn can be characterized by matrices, whose entries 'ij' encode information relating the connectivity of atoms or bonds ' $i$ ' and ' $j$ '. By manipulating these matrices with various mathematical algorithms, different types of topological and topochemical indices can be obtained. ${ }^{31}$ Among many others, the adjacency matrix is one of these matrices, actually the first which was introduced. Its construction for isopentane is illustrated in Figure 1.<smiles>CCC(C)C</smiles>

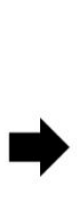

Isopentane

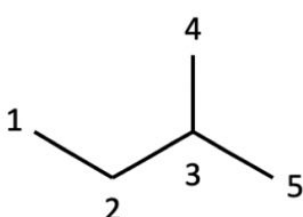

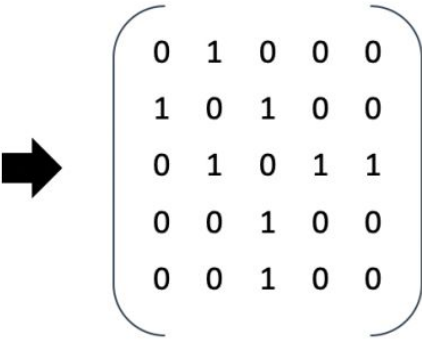

Adjacency matrix

Figure 1. Chemical representation, molecular graph and adjacency matrix for isopentane.

As far as we are aware, the present work is the first in which MT-QSAR is applied to the search of new SDA for the synthesis of zeolites. Other authors have used topological descriptors to model different properties related to zeolitic materials. For example, Torrens and Castellano used geometric and topological descriptors to calculate the fractal surfaces of crystals ${ }^{32}$ and Leflaive et 
al. employed topological descriptors to predict adsorption properties of molecules on different zeolites. ${ }^{33}$

The aim of this study is the identification of new organic compounds that may act as SDAs in the synthesis of BEA (polymorph A of Beta zeolite), BEC and ISV zeolites, which have important applications, including as acid catalysts for hydrocarbon conversions and the selective synthesis of fine chemicals. ${ }^{34}$ These zeolites have three-dimensional pore systems with 3-D intersecting channels formed by 12-membered rings and therefore possess analogous framework structures with similar pore topologies. Hence, it is not surprising that they compete in the synthesis process. ${ }^{35}$ Finding new SDAs for the synthesis of these zeolites in a more cost-efficient way than those already used is very relevant for industrial processes in which zeolites are employed.

In the present study, different computational techniques, including Monte Carlo simulations and energy minimization, have been used in combination with MT- QSAR such as. The goal was the identification of novel SDA candidates from a large database of organic molecules, as well as the development of quantitative energetic calculations to understand how the candidate SDAs interact with the zeolites under study.

\section{Computational methods}

\subsection{Selection of potential SDAs by molecular topology}

The first step to create a predictive model is to build, using information from the literature, a training set of molecules, specified below, that are active or inactive towards the synthesis of a particular zeolite. For BEA zeolite the study by Zones et al. ${ }^{36}$ provides 21 piperidinium candidate SDAs, of which 7 are active (lead to BEA) and 14 are inactive (do not lead to BEA). For BEC zeolite a total of 57 candidate SDAs, 15 active and 42 inactive, were selected from a large set of literature. ${ }^{2,3,37-44}$ For ISV, 21 molecules were selected as the training set for being candidate SDAs, with 8 active and 13 inactive, taken from publications in the group of Corma. ${ }^{3,35,45-48}$

Molecules forming the training set for all models are shown in Supporting Information (sections S2, S3 and S4). For all models reported here, the group of inactive compounds was composed of molecules that did not lead to the formation of the zeolite under study, although they could favor the synthesis of another zeolite. 'Inactive' molecules must therefore not be seen as useless since they may become 'active' for a different target. Scheme 1 shows all the computational methods employed in this study. 


\subsection{Molecular descriptors}

Topological indices, as other approaches based on fingerprints, codify information about molecular structure in a purely numerical way. Their numerical format notably simplifies the automatic search of other molecules with similar structural properties, hence they are strong candidates to share the physicochemical and biological properties sought ${ }^{49}$

Each compound was characterized by a set of 436 topological indices. All indices were calculated with Dragon software. ${ }^{50}$ The topological indices together to their values for the molecules conforming the models are given as Supporting Information (sections S2, S3 and S4).

\subsection{Modeling techniques and quality model parameters.}

The statistical technique used for the construction of the models presented here is the linear discriminant analysis (LDA) ${ }^{51}$ as implemented in the STATISTICA software. ${ }^{52}$ LDA is a supervised pattern recognition method which provides a classification model based on the combination of variables that best predict the category or group to which a given compound belongs; in our case, whether -or not- the molecule presented, described by several topological indices, acts as a structure-directing agent towards BEA, BEC or ISV zeolite synthesis. The capability of acting as SDA to synthesize BEA, BEC and ISV was chosen as discriminant 
property, with the independent variables being the topological indices. The same weight (0.5) was assigned to the group of active and inactive molecules regardless of the number of compounds belonging to these two categories.

The algorithm proceeds iteratively. The method used to select the descriptors is based on a coefficient ( $p$ ) that determines the relative importance of candidate variables. The variables used to compute the linear classification function are chosen in a stepwise manner depending on the value of ' $p$ ': at each step, the variable that makes the largest ' $p$ ' contribution to the separation of the groups is entered into the discriminant equation (or the variable that makes the smallest ' $p$ ' contribution is removed). From initially 436 descriptors, a preselection of the most important descriptors was performed. After this preselection, a discriminant function (DF) is specified that contains a linear combination of the selected descriptors. For each molecule, activity is assessed by $\mathrm{DF}>0$ and inactivity by $\mathrm{DF}<0$.

\subsubsection{Wilks parameter ( $\lambda$ )}

The goodness of the model, called the 'discriminant capability', was evaluated using the Wilks parameter, $0 \leq \lambda \leq 1$, which was obtained by multivariate analysis of variance that tests the equality of group average for the variable in the discriminant model. The Wilks' lambda parameter measures the ability of predictive model variables to discriminate. Higher values mean less discriminant information inside the variables and therefore less difference between groups. ${ }^{53}$ Values of $\lambda \leq 0.6$ are considered as having exceeded the threshold of good quality (see test \#1 in Scheme 1)..$^{53}$

\subsubsection{Confusion matrix and Matthews Correlation Coefficient (MCC)}

From the DF values obtained for each molecule, and depending on whether they are or not truly active and inactive, four possibilities arise that are compiled in the so called confusion matrix: correct predictions are TP (true positive) and TN (true negative), whilst incorrect predictions can be an active detected as inactive (FN, false negative) or an inactive detected as active (FP, false positive). The percentage of correct actives $(\mathrm{TP} /(\mathrm{TP}+\mathrm{FN}) \times 100)$ and correct inactives $(\mathrm{TN} /(\mathrm{TN}+\mathrm{FP}) \times 100)$ is one test of the goodness of the model (see test \#2 in Scheme 1).

The Matthews correlation coefficient (MCC) ${ }^{54}$ is a parameter that provides a balanced evaluation of the prediction. This coefficient is based on the fact that, in any prediction process, four different possibilities can occur: TP (true positive), active compounds correctly classified (SDA for BEA, BEC or ISV); FP (false positive), inactive compounds classified as active; TN (true negative), inactive compounds correctly classified, and FN (false negative), active compounds classified as inactive. MCC is then defined as follows.

$$
M C C=\frac{T P \times T N-F P \times F N}{\sqrt{(T N+F N) \times(T N+F P) \times(T P+F P) \times(T P+F N)}}
$$

The Matthews correlation coefficient ranges from $-1 \leq \mathrm{MCC} \leq 1$. A value of $\mathrm{MCC}=1$ indicates a perfect prediction, in which every compound in the model was correctly labeled, whereas 
$\mathrm{MCC}=-1$ (anti-correlation) means that not even one single molecule was correctly classified. MCC can also be redefined on a percent basis as $\mathrm{MCC}(\%)=(\mathrm{MCC}+1) \times 50$.

\subsubsection{Receiver Operating Characteristic (ROC) curve}

Success and error observed in a classification model can be plotted by the receiver operating characteristic (ROC) curve ${ }^{55}$ (see test \#3 in Scheme 1). The ROC curve evaluates the accuracy of the DFs, selected through the sensitivity (true positive fraction) and specificity (true negative fraction) for different thresholds of the discriminant function. Hence, the ROC curve represents sensitivity versus 1 -specificity (false positive fraction). The closer the curve follows the left hand border and then the top border of the ROC space, the more accurate is the model. The closer the curve comes to the $45^{\circ}$ diagonal of the ROC space, the less accurate is the test. A completely random classification would give a plot along the diagonal line, which is also called a nondiscrimination line. ${ }^{56}$ Accuracy is measured by the area under the ROC curve (AUC). ${ }^{57}$ An area of 1 represents a perfect test; and an area of 0.5 (or lower) represents a worthless test. In general, AUC values $>0.9$ are considered very good, and values $>0.97$ are excellent.

\subsection{Validation of the modeling techniques}

Once the prediction model is built, the validation of the model can be performed on two types of data: data that was used in the construction of the model (internal validation) and data that was not used in the construction (external validation). The internal validation allows analyzing the goodness of the model, and the external validation allows to know if the predictive performance of the model declines when applied to a new data. ${ }^{58}$

In our particular case, we selected the internal validation techniques, because our data is not very abundant. Internal validation can be performed by testing the prediction of one or more elements left out in a training set of the other elements. ${ }^{59}$ This approach is the so called leave-one-out (LOOCV) or leave-some-out (LSOCV) cross-validation. In the particular case of LOOCV, every element in the training set has been tested. At the end of LOOCV, the classification ability of the discriminant function can be evaluated by recalculation of the Wilks parameter $\left(\lambda^{\prime}\right)$ in each if the LOO cases. This gives as many values as molecules in the training set, and the average value of $\lambda^{\prime}$ is given as a final result. Similar values of $\lambda$ and $\lambda^{\prime}$ indicates a good validation of the model. This is also shown in Scheme 1 (internal validation \#4). The same procedure is used for LSOCV, but instead of choosing one element of the test set each step, a group of elements acts as test set in a stepwise manner. Specifically, $25 \%$ of the data set was chosen randomly to act as test set and the procedure was repeated ten times.

\subsection{Applicability domain of the models: Activity distribution diagram (ADD)}

An activity distribution diagram (ADD) is a graphical representation that provides a straightforward way of visualizing the regions of minimum overlap between active and inactive compounds, as well as the regions in which the probability of finding active compounds is at a maximum. ${ }^{60}$ In fact the ADD is a frequency distribution diagram of dependent variables in which the ordinate represents the expectancy (probability of activity) and the abscissa represents the DF values in the range. For an arbitrary range of values of a given function, an "expectancy of 
activity" can be defined as $E_{a}=a /(i+1)$, where " $a$ " is the number of active compounds in the range (TP+FN in the range) divided by the total number of active compounds (total TP+FN), and " $i$ " is the number of inactive compounds in the interval (TN+FP in the interval) divided by the total number of inactive compounds (total $\mathrm{TN}+\mathrm{FP}$ ). Expectancy of activity $\left(\mathrm{E}_{\mathrm{a}}\right)$ is shown as \#5 in Scheme 1 . The expectancy of inactivity is defined in a symmetrical way as $E_{i}=i /(a+1)$. Presented with these diagrams, it is easy to visualize the intervals in which there is a maximum probability of finding new active compounds and a minimum probability of finding inactive compounds. This graph gives information about the range of applicability of the model.

\subsection{Virtual screening of chemical libraries}

Once the models are validated, a virtual screening of commercially available database compounds has been performed (see \#6 in Scheme 1). Databases from Sigma-Aldrich obtained from the ZINC server, ${ }^{61}$ Specs,${ }^{62}$ Asinex,${ }^{63}$ and eMolecules ${ }^{64}$ have been screened in order to select potential novel structure-directing agents for the synthesis of BEA, BEC and ISV zeolites. The total number of molecules included for screening is over 7 million compounds.

\subsection{Monte Carlo and lattice energy minimization calculation of zeolite-SDA interactions}

The usual assumption, from the computational chemistry perspective, is that the zeolite-template interaction energy is a valid approximation that can be applied. ${ }^{65}$ The lower the energy the larger the stability. In a further refinement, we suggested the stabilisation energy divided per $\mathrm{SiO}_{2}$, that includes the concept of loading and the porosity of the zeolite. ${ }^{66}$ Both energies are considered important: the energy per SDA molecule (for which only one SDA molecule needs to be introduced in the zeolite unit cell), and the energy per $\mathrm{SiO}_{2}$ (which requires the unit cell to be filled with as many SDA molecules as possible). The energetic stabilisation can be calculated by including a number of contributions such as the stability of the zeolite and the effect of the template counteranion, but the most important term is always the dispersion interaction between zeolite and template, which is the only one that will be included in the present study. The zeoTsda ${ }^{25}$ software incorporates a combination of Monte Carlo and lattice energy minimization as calculated by GULP ${ }^{67}$ with an interface that takes into account information on the system in order to take decisions on whether or not the inclusion of more SDA molecules into the system will contribute to make the new system more stable. The software allows the procedure to be automated for one or two databases (templates and/or zeolites) acting simultaneously in order to calculate all the zeolite-SDA combinations sequentially.

Our approach follows the simplified scheme of our previous publication ${ }^{25}$, in which we consider a pure silica zeolite structure with a neutralised SDA. The latter means overall zero charge, so that the summation of all atomic charges gives a neutral molecule. This avoids the inclusion of $\mathrm{Al}$, inorganic cations, and/or counteranions (hydroxide, fluoride) that will increase the complexity of each calculation as well as the number of Al-distributions that may need to be considered. We analyze the results focusing on the most important zeo-SDA dispersion contribution, but the calculations have been performed by considering and minimizing all the energetic interactions, 
including electrostatics. Future versions of our software will increase complexity and accuracy. In the current version the predictions can only be made for pure silica zeolites.

\section{Results and discussion}

\subsection{Candidate SDAs for BEA zeolite}

A prediction model of piperidine compounds showing activity as structure-directing agents towards the synthesis of BEA zeolite is presented. The model $\left(\mathrm{DF}_{\mathrm{BEA}}\right)$ consists of two descriptors as seen in equation 1 :

$$
\begin{aligned}
& D F_{B E A}=1.01+(15.87 \times S 3 K)-(10.41 \times \text { EEig } 05 d) \\
& \quad \mathrm{N}=21 ; \lambda=0.468 ; \mathrm{F}=10.213 ; \mathrm{p}<0.001
\end{aligned}
$$

Where $\mathrm{N}$ is the number of training set compounds, $\lambda$ is the Wilks parameter, $\mathrm{F}$ is the FisherSnedecor parameter and $\mathrm{p}$ is the statistical significance. The Fisher-Snedecor parameter gives us information on how significant the descriptors selected in the model are. Whereas the p-value shows us the probability of having obtained the result, if we assume that the null hypothesis is true. That is, if the value of $p$ is lower than the level of significance (conventionally 0.05 or 0.01 ), it is possible to test the null hypothesis that the multivariate means of the groups (active and inactive) are equal.

The descriptors chosen are: S3K (3-path Kier alpha-modified shape index -topological index) and EEig05d (eigenvalue n. 5 from augmented edge adjacency matrix weighted by dipole moment edge adjacency index). A detailed analysis of the meaning and importance of these descriptors is included as Supporting Information (section S1).

All training set compounds are reported in section S2 in the Supporting Information, showing: probability of activity, DF value and value of each respective descriptor contributing to the discriminant equation. A molecule will be classified, according to the result of $\mathrm{DF}_{\mathrm{BEA}}$ as potential $\mathrm{SDA}$ for $\mathrm{BEA}$ if $\mathrm{DF}>0$, and inactive if $\mathrm{DF}<0$. A range of $\mathrm{DF}$ values for activity and inactivity will be obtained, without implying that the higher (lower) the DF value the higher (lower) the activity (inactivity). By applying this criterion to the training set of 21 compounds, 6 out of 7 were correctly classified as active ( $86 \%$ accuracy) while 14 out of the 14 inactive compounds were correctly classified as inactive (Table 1 ).

As can be noticed in Table 1, results derived from the classification matrix showed that this model (Eq. 1) presents a greater specificity than sensitivity. This is ideal for applying the model to a virtual screening of compounds with the aim of identifying new SDAs that direct the synthesis towards the crystallization of BEA zeolite. In this way, the probability of finding false active (FP, false positive) SDAs will be lower. Although we could identify some SDAs misclassified as inactive (false negative, FN), this will not be relevant since we are only interested in selecting active SDAs (TP+FP). 
Table 1. Classification matrix (also called confusion matrix) obtained through the calculation using the discriminant functions $\mathrm{DF}_{\mathrm{BEA}}, \mathrm{DF}_{\mathrm{BEC}}$, and $\mathrm{DF}_{\mathrm{ISV}}$ for the respective training sets. Compounds correctly classified as active and inactive are also called TP (true positive) and TN (true negative) respectively. Compounds incorrectly classified are an active detected as inactive (FN, false negative) or an inactive detected as active (FP, false positive).

\begin{tabular}{cccc} 
& $\begin{array}{c}\% \text { Correct } \\
\text { classification }\end{array}$ & $\begin{array}{c}\mathbf{N}^{\mathbf{0}} \text { compounds } \\
\text { class. as Active }\end{array}$ & $\begin{array}{c}\mathbf{N}^{\mathbf{0}} \text { compounds } \\
\text { class. as Inactive }\end{array}$ \\
\hline Training set for BEA & & & \\
\hline Active group & 86 & 6 & 1 \\
Inactive group & 100 & 0 & 14 \\
\hline Training set for BEC & & & 2 \\
\hline Active group & 87 & 13 & 36 \\
Inactive group & 86 & 6 & 1 \\
\hline Training set for ISV & & & 11 \\
\hline Active group & 88 & 7 & \\
Inactive group & 92 & 1 & \\
\hline
\end{tabular}

Table 1 shows that the model relies on a very small data set, which constitutes a limitation when validating it. In spite of this, the Matthews correlation coefficient (MCC), gives the value 0.894 , therefore giving a 95\% accuracy for the model. The ROC curve, which gives a visual illustration of the success and error observed in a classification model, is outlined in Figure 2. The area under the curve (AUC) from $\mathrm{DF}_{\mathrm{BEA}}$ is 1 , which means a perfect separation of the values of the two groups, i.e. there is no overlap of the distributions.

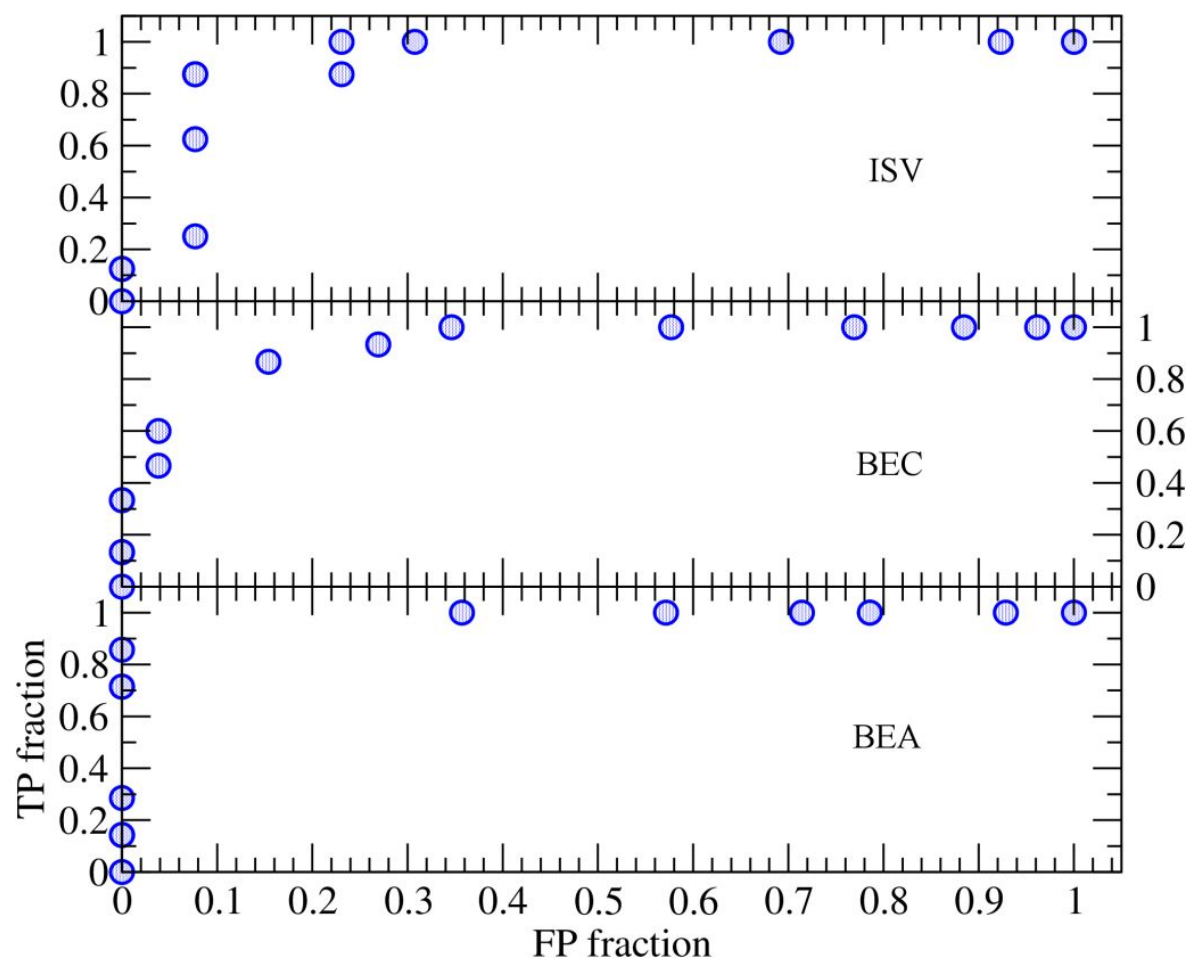

Figure 2. $R O C$ (receiver operation characteristic) curves for $\mathrm{DF}_{\mathrm{BEA}}, \mathrm{DF}_{\mathrm{BEC}}$ and $\mathrm{DF}_{\mathrm{ISV}}$, showing the fractions of: true positives (vertical axis) and false positives (horizontal axis). 
Finally, an internal validation of the model was performed. A cross-validation following the leave-one-out and leave-some-out techniques yielded an average value of $\lambda=0.465$ for LOOCV and $\lambda=0.463$ for LSOCV, which is almost the same value obtained from $\mathrm{DF}_{\mathrm{BEA}}: \lambda=0.468$, meaning that the model is consistent. Section S2 in the Supporting Information shows more details of the LOOCV and LSOCV calculations.

In order to test the applicability domain, the activity distribution diagram (ADD) has been plotted (Figure 3). This shows that compounds active towards the synthesis of BEA zeolite adopt $\mathrm{DF}_{\mathrm{BEA}}$ values between $0-7$, although an overlap zone (between [-1, 0.5]) is appreciated. Compounds that do not direct towards the formation of $\mathrm{BEA}$ zeolite adopt negative values in the $\mathrm{DF}_{\mathrm{BEA}}$ equation, ranging from 0 to -7 . Compounds that have values of $\mathrm{DF}_{\mathrm{BEA}}>7$ or $<-7$ will be regarded as not classifiable (NC) by our model.

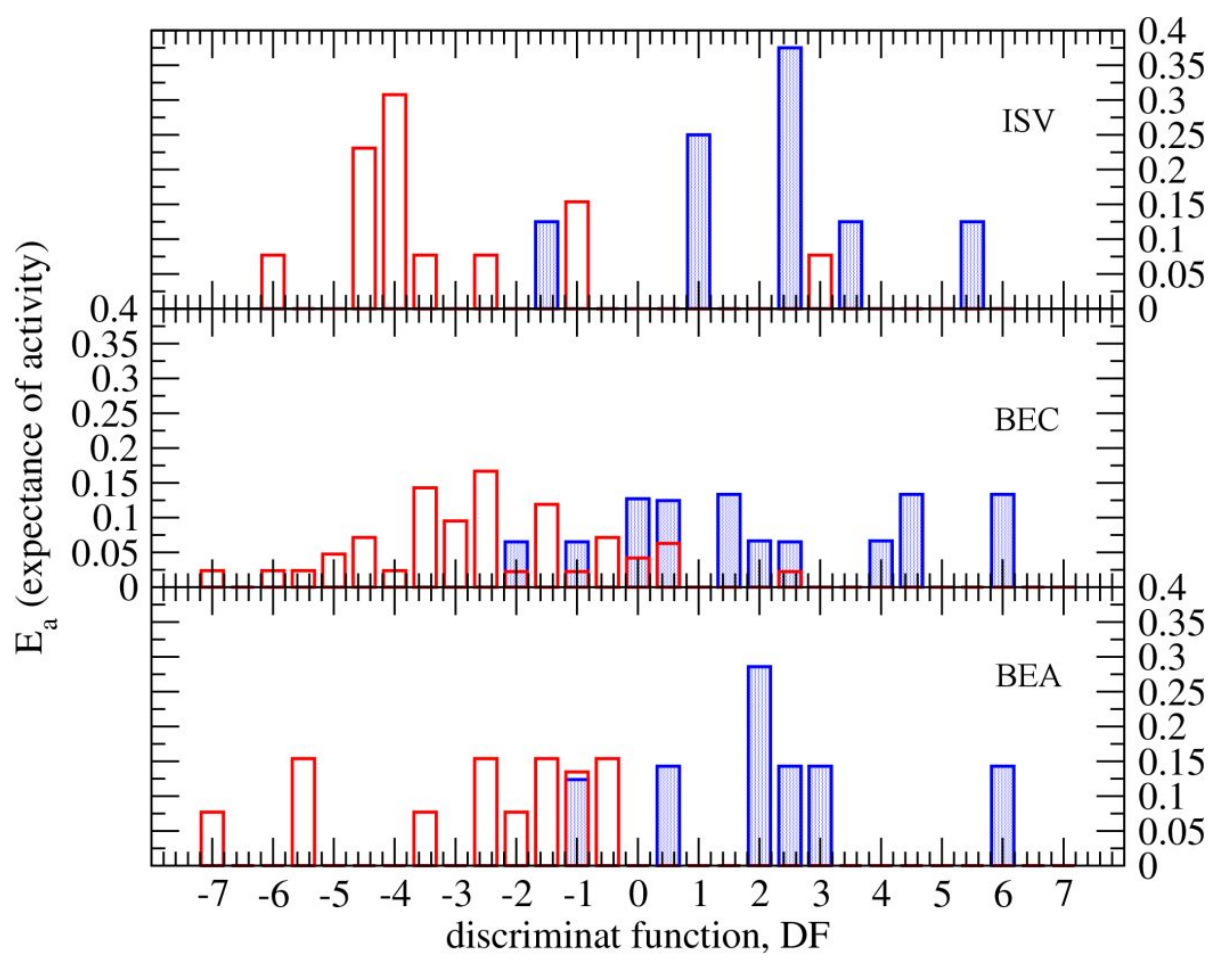

Figure 3. Activity distribution diagram (ADD) for $\mathrm{DF}_{\mathrm{BEA}}, \mathrm{DF}_{\mathrm{BEC}}$ and $\mathrm{DF}_{\mathrm{ISV}}$. Showing the expectancy of activity $\left(E_{a}\right.$, as defined in $\left.\S 2.5\right)$ versus the discriminant function, $D F$ (see section $\S 2.3$ and equations 1-3). Blue and red bars indicate activity and inactivity respectively.

\subsection{Candidate SDAs for BEC zeolite}

The prediction model for SDAs that direct the formation of ITQ-17 (BEC) zeolite rendered the following discriminant function, $\mathrm{DF}_{\mathrm{BEC}}$ :

$D F_{2}=(4.880 \times I C R)+(0.174 \times p i I D)-(16.516 \times I D D M)+(11.048 \times M A T S 3 m)$

$+43.101$

(Eq. 2) 


$$
\mathrm{N}=57 ; \lambda=0.526 ; \mathrm{F}=11.721 ; \mathrm{p}<0.00001
$$

The descriptors chosen were: ICR (radial centric information index-topological index), piID (conventional bond order ID number -walk and path counts), IDDM (mean information content on the distance degree magnitude-information index), and MATS3m (Moran autocorrelation of lag 3 weighted by atomic masses - $2 \mathrm{~d}$ autocorrelations). A detailed analysis of the meaning, importance and the actual values of these descriptors is included as Supporting Information (section S1).

A molecule will be classified according to the result of $\mathrm{DF}_{\mathrm{BEC}}$ as potential (active) SDA for the synthesis of $\mathrm{BEC}$ if $\mathrm{DF}_{\mathrm{BEC}}>0$, and inactive if $\mathrm{DF}_{\mathrm{BEC}}<0$. By applying this criterion to the training set of 57 compounds, 13 out of 15 were correctly classified ( $87 \%$ accuracy) while 36 out of 42 inactive compounds were correctly classified ( $86 \%$ accuracy), as can be seen in Table 1 .

In this case, $\mathrm{DF}_{\mathrm{BEC}}$ is a model as specific as it is sensitive. So there is a similar probability of finding false active $(\mathrm{FP} /(\mathrm{FP}+\mathrm{TN}) \times 100)$ and false inactive $(\mathrm{FN} /(\mathrm{FN}+\mathrm{TP}) \times 100)$ when applying the model $(\approx 14 \%)$.

The MCC parameter gives a value of 0.676 , yielding an $84 \%$ of accuracy for the model. The AUC corresponding to the ROC curve (Figure 2) has a value of 0.956 , which means that the model has a $96 \%$ probability that the compound selected as active is truly active (TP) compared to the possibility that it is a false positive. This means the number of false positives is very small, indicating a good quality model.

A leave-one-out and leave-some-out cross-validation techniques have been performed yielding an average value of $\lambda=0.525$ (LOOCV) and $\lambda=0.543$ (LSOCV), which is almost the same discriminant rate as the original value for the model, $\lambda=0.526$. Again, values below 0.6 indicate a reasonable quality. More details are included as Supporting Information (section S3).

In Figure 3, the activity distribution diagram (ADD) shows the applicability domain of $\mathrm{DF}_{\mathrm{BEC}}$. This indicates that compounds that lead to the synthesis of $\mathrm{BEC}$ zeolite adopt $\mathrm{DF}_{\mathrm{BEC}}$ values between 0-7, although there is an overlapping zone between $\mathrm{DF}_{\mathrm{BEC}}$ values from -2 to 3 . Compounds that do not direct towards BEC formation mostly adopt negative values in the equation, with $\mathrm{DF}_{\mathrm{BEC}}$ values between 0 and -7 . Compounds having $\mathrm{DF}_{\mathrm{BEC}}$ values $>7$ or $<-7$ will be regarded as not classifiable by the model as it falls outside its range of applicability.

\subsection{Candidate SDAs for ISV zeolite}

A model for the prediction of compounds acting as SDAs to the formation of ITQ-7 (ISV) zeolite has been achieved. The following discriminant function has been found (Eq. 3):

$$
\begin{aligned}
& D F_{I S V}=10.43+(0.23 \times D D)-(19.83 \times P C R) \\
& \mathrm{N}=20 ; \lambda=0.418 ; \mathrm{F}=12.506 ; \mathrm{p}<0.0004
\end{aligned}
$$


The parameters accounting for the significance of this equation were: DD (distance/detour matrix - topological descriptors); and PCR (ratio of multiple path count over path count - walk and path counts descriptors). More details about them are given in the Supporting Information (section S1).

A molecule will be classified, according to the result of $\mathrm{DF}_{\mathrm{ISV}}$, as potential SDA if $\mathrm{DF}>0$, and inactive if $\mathrm{DF}<0$. By applying this criterion to the training set of 21 compounds, 7 out of 8 were correctly classified as active ( $88 \%$ accuracy) while 11 out of 12 inactive compounds were correctly classified ( $92 \%$ accuracy), as can be seen in Table 1 .

The data of ISV in Table 1 is indicative of a model with greater specificity than sensitivity. This is ideal for application to a virtual screening of compounds with the aim of identifying new SDAs that direct the synthesis towards the formation of ISV zeolite. In this way, the probability of finding false SDAs is expected to be low. Although we could miss the identification of some valid SDAs, this model will rarely classify as active an inactive molecule, hence the number of FN (false negatives) will be small.

The MCC value for $\mathrm{DF}_{\text {ISv }}$ is 0.792 , therefore with a $90 \%$ accuracy, and the value for AUC is 0.923 (Figure 2), which means the model has a $92 \%$ probability that the compound selected as active is a truly active. Leave-one-out (LOOCV) technique yields an average value of $\lambda=0.417$ and the leave-some-out approach (LSOCV), yields an average value of 0.408 which is also close as the original model $(\lambda=0.418)$. Section S4 in the Supporting Information gives more details.

As noticed from the ADD curve (Figure 3), compounds that direct towards ISV zeolite give DF ISV

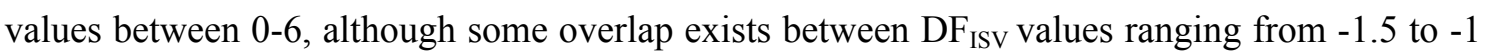
and from 3 to 3.5. Compounds that do not direct towards ISV display negative values in the equation ranging from 0 to -6 . Finally, compounds with values of $\mathrm{DF}_{\mathrm{ISV}}>6$ or $<-6$ will be regarded as not classifiable by the model.

\subsection{Virtual screening. Identifying novel SDA for the synthesis of BEA, BEC and ISV}

From the above study, a preselection of 29 candidate SDAs was obtained (section S5 from Supporting information). This preselection includes all candidate SDAs clearly identified by our topological models as active for the synthesis of BEA, BEC or ISV zeolite. The following step is to assess accurately, from an atomistic viewpoint, how good they might be as templates. The results are summarized in Table 2, where the dispersion zeolite-SDA energy is indicated for the maximum loading calculated by zeoTsda and divided by the number of $\mathrm{SiO}_{2}$ units in the unit cell. The unit cells selected for the calculations of the three zeolites (BEA, BEC, ISV) contain the same number of atoms $\left(64 \mathrm{SiO}_{2}\right)$. Table 2 also contains the values for the discriminant function, which allows comparing the predictions of the topological model with the zeolite-SDA dispersion energy. The values highlighted indicate a preferential energy (by $>0.6 \mathrm{~kJ} / \mathrm{mol} \mathrm{Si}$ ) to stabilize a particular zeolite structure with the SDA. In our previous study ${ }^{10}$ we suggested that not only a low value of E(zeolite-SDA) is needed, but also -for the sake of selectivity- a considerably larger stability in one zeolite over the other competing phases. 
Table 2. Dispersion zeolite-SDA energya, E(z-S) in $\mathrm{kJ} / \mathrm{mol} \mathrm{Si}$, for the templates preselected according to the values of the discriminant function (DF), at the maximum loading (molecules/unit cell), calculated using zeoTsda software, for BEA, BEC and ISV zeolites.

\begin{tabular}{|c|c|c|c|c|c|c|c|c|c|c|}
\hline \multirow[b]{2}{*}{ SDA } & \multirow[b]{2}{*}{$\mathbf{D F}_{\mathrm{BEA}}$} & \multirow[b]{2}{*}{$\mathbf{D} \mathbf{F}_{\mathrm{BEC}}$} & \multirow[b]{2}{*}{$\mathrm{DF}_{\text {ISV }}$} & \multicolumn{2}{|r|}{ BEA } & \multicolumn{2}{|c|}{ BEC } & \multicolumn{2}{|r|}{ ISV } & \multirow[b]{2}{*}{ Guess } \\
\hline & & & & $\begin{array}{l}\text { load } \\
\mathrm{m} / \mathrm{uc}\end{array}$ & $\begin{array}{c}\mathrm{E}(\mathrm{z}-\mathrm{S}) \\
(\mathrm{kJ} / \mathrm{molSi})\end{array}$ & $\begin{array}{l}\text { load } \\
\mathrm{m} / \mathrm{uc}\end{array}$ & $\begin{array}{c}\mathrm{E}(\mathrm{z}-\mathrm{S}) \\
(\mathrm{kJ} / \mathrm{molSi})\end{array}$ & $\begin{array}{l}\text { load } \\
\text { m/uc }\end{array}$ & $\begin{array}{c}\mathrm{E}(\mathrm{z}-\mathrm{S}) \\
(\mathrm{kJ} / \mathrm{molSi})\end{array}$ & \\
\hline SDA1 & 23.0 & 1.3 & -3.5 & 4 & -4.52 & 4 & -5.27 & 4 & -4.37 & $\mathrm{BEC}$ \\
\hline SDA2 & -15.9 & 1.0 & 4.7 & 4 & -6.33 & 2 & -3.92 & 3 & -6.18 & \\
\hline SDA3 & 13.1 & 5.6 & 4.0 & 4 & -7.23 & 4 & -6.48 & 3 & -5.72 & BEA \\
\hline SDA4 & -11.6 & -2.6 & 0.1 & 4 & -6.48 & 4 & -6.33 & 4 & -6.93 & \\
\hline SDA5 & -6.6 & -1.4 & 0.6 & 4 & -7.99 & 4 & -6.63 & 4 & -6.03 & BEA \\
\hline SDA6 & 0.7 & -1.8 & 0.2 & 4 & -7.69 & 4 & -3.47 & 4 & -6.63 & BEA \\
\hline SDA7 & 5.2 & 4.4 & 4.5 & 4 & -4.22 & 3 & -5.12 & 4 & -7.23 & ISV \\
\hline SDA8 & -15.4 & 2.8 & -0.9 & 4 & -7.54 & 5 & -3.92 & 4 & -7.99 & \\
\hline SDA9 & 19.8 & 4.3 & 22.8 & 4 & -7.69 & 3 & -5.57 & 3 & -5.72 & BEA \\
\hline SDA10 & 11.5 & 1.9 & 6.0 & 4 & -6.18 & 4 & -7.38 & 4 & -7.38 & \\
\hline SDA11 & 5.3 & 9.0 & 1.3 & 4 & -5.87 & 4 & -5.42 & 4 & -3.02 & \\
\hline SDA12 & 5.1 & 6.9 & 3.2 & 4 & -6.03 & 4 & -6.03 & 3 & -4.67 & \\
\hline SDA13 & 15.3 & 1.2 & 2.3 & 4 & -5.42 & 4 & -5.72 & 4 & -5.27 & \\
\hline SDA14 & 17.0 & -3.4 & 1.4 & 4 & -5.87 & 4 & -4.67 & 4 & -6.48 & ISV \\
\hline SDA15 & 15.4 & -1.2 & 5.9 & 3 & -5.42 & 3 & -4.67 & 3 & -5.12 & \\
\hline SDA16 & 5.7 & 2.6 & 5.4 & 4 & -6.63 & 4 & -6.93 & 4 & -6.03 & \\
\hline SDA17 & 1.3 & -0.4 & 5.3 & 4 & -6.63 & 5 & -6.03 & 4 & -6.03 & BEA \\
\hline SDA18 & 3.0 & 0.0 & 6.7 & 4 & -6.48 & 4 & -5.72 & 4 & -6.03 & \\
\hline SDA19 & 4.8 & 0.2 & 5.8 & 4 & -8.29 & 4 & -7.38 & 3 & -6.18 & BEA \\
\hline SDA20 & 5.6 & 5.3 & 4.3 & 3 & -5.12 & 4 & -7.54 & 4 & -6.63 & $\mathrm{BEC}$ \\
\hline SDA21 & 5.2 & 4.8 & 5.4 & 3 & -5.72 & 3 & -4.97 & 3 & -4.52 & \\
\hline SDA22 & 14.5 & 5.1 & 7.6 & 3 & -5.42 & 4 & -7.23 & 4 & -5.87 & BEC \\
\hline SDA23 & 7.1 & -1.1 & 5.6 & 3 & -4.37 & 3 & -5.57 & 3 & -5.12 & \\
\hline SDA24 & 13.4 & -1.3 & 2.6 & 3 & -5.42 & 3 & -4.67 & 4 & -6.18 & ISV \\
\hline SDA25 & 15.1 & -0.6 & 5.9 & 3 & -5.27 & 2 & -3.17 & 3 & -5.42 & \\
\hline SDA26 & 6.5 & 3.6 & 5.6 & 3 & -5.12 & 4 & -5.12 & 4 & -6.48 & ISV \\
\hline SDA27 & 3.8 & 1.6 & 7.7 & 3 & -5.42 & 3 & -5.12 & 2 & -5.27 & \\
\hline SDA28 & 2.8 & 6.6 & 16.2 & 3 & -5.57 & 3 & -5.72 & 3 & -5.57 & \\
\hline SDA29 & 0.0 & 3.4 & 8.2 & 2 & -5.72 & 2 & -3.62 & 3 & -5.27 & \\
\hline
\end{tabular}

m.:molecule; u.c.:unit cell

${ }^{a}$ Energies that stabilize a structure over the others by $>0.6 \mathrm{~kJ} / \mathrm{mol} \mathrm{Si}$ are highlighted in grey, leading to the corresponding guess in the zeolite phase that might be obtained using the SDA. SDA figures are given as Supporting Information (section S7). 

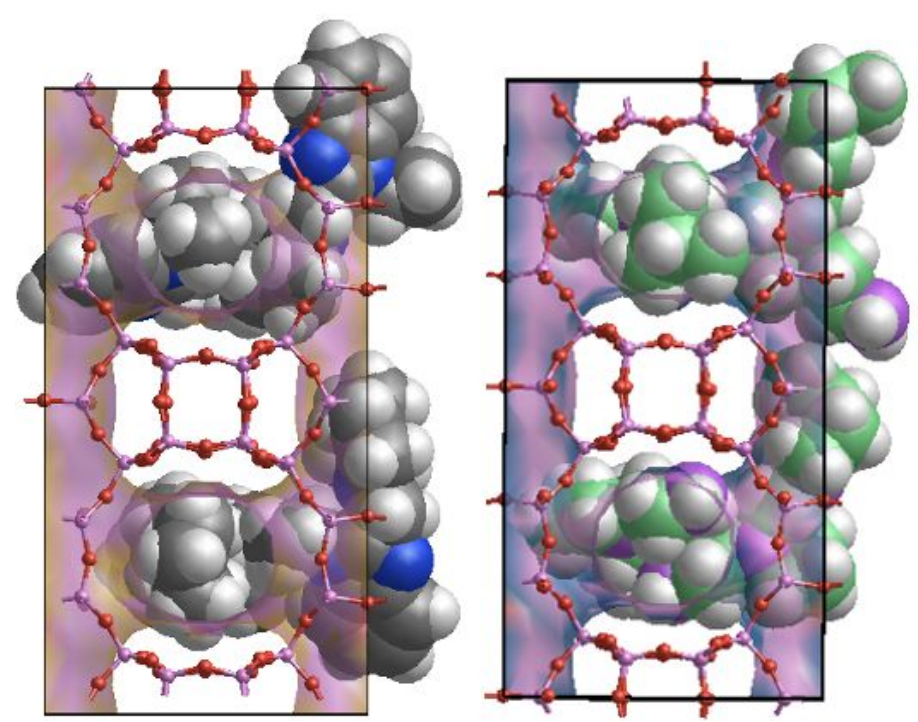

Figure 4. Optimized location of SDA20 (left) and SDA22 (right) in BEC zeolite with a loading of 4 molecules per unit cell. BEC micropore system is shadowed and partially transparent.

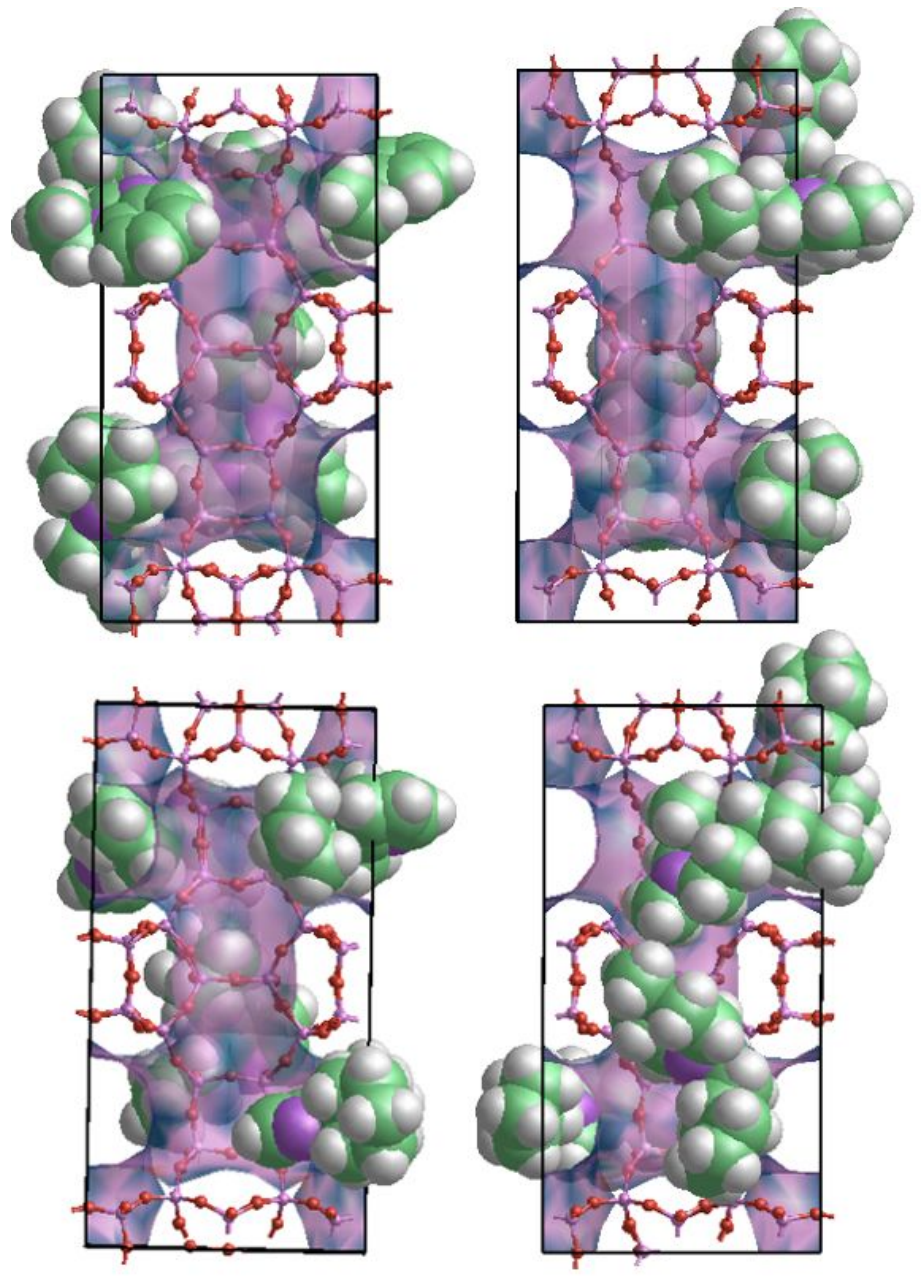

Figure 5. Optimized location of SDA7 (top left), SDA14 (top right), SDA24 (bottom left) and SDA26 (bottom right) in ISV zeolite with a loading of 4 molecules per unit cell. ISV micropore system is shadowed and partially transparent. 
According to these criteria, 13 SDAs have been highlighted (Table 2) that we consider candidates to synthesize BEA (6 SDAs), BEC (3 SDAs) and ISV (4 SDAs). In all selected cases, the optimum loading found is 4 SDA molecules per unit cell $\left(64 \mathrm{SiO}_{2}\right)$ (see section $\mathrm{S} 8$ from Supporting information), and this is in agreement with the pore size topology where at least 4 channel intersections are found in the unit cells. However, small molecules have also been found to be active templates giving a loading of 6 molecules per unit cell (more information on section S6 from Supporting information). ${ }^{17}$

In our current strategy of using large SDA molecules, with an optimum loading of 4 molecules per unit cell, SDA5 and SDA19 are perhaps the most appropriate for BEA due to their strong stabilization energies (-7.99 and -8.29 kJ/mol Si respectively, see Table 2). However, since BEA is the most popular and widely used of these three zeolites, it is certainly of most interest to focus on templates that allow the synthesis of BEC and ISV.

From the three SDAs suggested for BEC, SDA1 can be discarded as it gives a relatively weak stabilization energy $(-5.27 \mathrm{~kJ} / \mathrm{mol} \mathrm{Si})$, due to its relatively small size. Being an almost linear molecule, SDA1 cannot adapt its shape to the channel intersections and hence it does not seem to be a good candidate. The other two candidates, SDA20 and SDA22 show a perfect fit in the pore size of BEC as shown in Figure 4, and their respective zeo-SDA dispersion energies (-7.54 and $7.23 \mathrm{~kJ} / \mathrm{mol} \mathrm{Si}$, Table 2) are considerably low, indicating high stability.

For ISV, four SDAs seem appropriate, SDA7, SDA14, SDA24 and SDA26, whose excellent match inside the pore system is shown in Figure 5. Their zeo-SDA energies (-7.23, -6.48, -6.18 and $-6.48 \mathrm{~kJ} / \mathrm{mol} \mathrm{Si}$ respectively) are not as low as in the previous cases for BEA and BEC, but they are clearly lower than those for the competing BEA and BEC zeolites by more than 0.6 $\mathrm{kJ} / \mathrm{mol} \mathrm{Si}$.

It is not easy to justify the fluctuations observed in the maximum uptake of the different templates in Table 2. For templates of different sizes, the differences in full loading conform logically to their different maximum uptakes. For templates of similar sizes, differences can be explained by two reasons: a) sometimes small differences in template geometry make a significant difference in loading when template size is matching the micropore topology; b) the Monte Carlo algorithm, that is based on random numbers (stochastic algorithms), is sometimes not able to find the best location of the template in the zeolite micropore. Several parameters have been tuned and modified systematically; not only the number of cycles, but also the ratio between rotation/translation moves, in order to ensure the quality of the results. In the end, we cannot identify which of these two reasons is responsible for the results.

An analysis of results from the topological model predictions (discriminant function, DF, columns in Table 2) shows, in the first place, that none of the 30 molecules preselected is a bad template according to the E(zeo-SDA) energies- with, perhaps, the exception only of SDA1. A few differences can be observed in general with respect to the results of E(zeo-SDA).

SDA20 and SDA22, selected as potentially good candidates to synthesize BEC do show positive values of $\mathrm{DF}_{\mathrm{BEC}}$, showing agreement between both predictions, albeit the $\mathrm{DF}_{\mathrm{BEC}}$ values are not 
particularly high. SDA7, SDA14, SDA24 and SDA26, predicted from E(zeo-SDA) as potential templates for the synthesis of ISV, show positive values of $\mathrm{DF}_{\text {ISv }}$.

The numerical values of DF are not so important because the topological models for BEA, BEC and ISV were considered independently, and therefore without introducing the fact that one template defined as active for one zeolite should have been defined as inactive for the other two. It could be seen that all selected SDAs, except for SDA5, show corroboration between Monte Carlo simulation and DF value. In other words, selected SDAs for BEA zeolite are classified as active by $\mathrm{DF}_{\mathrm{BEA}}$ models, as do those of BEC and ISV. Hence, there is consistency between the results obtained using MT-QSAR that do not account for 3-D aspects and those that are derived from the Monte Carlo methods (3-D taken into account). However, there a not a direct correlation between DF and E(z-S).

The current model was designed with the main purpose (first objective) of discarding bad templates from the final preselection and this objective has been achieved. Although a few templates were selected as candidates for selective synthesis, this second objective has proven much more elusive, especially given the fact that the three zeolites have very similar microporous systems. This remains a challenge for future work.

\section{Conclusions}

A model based on topological descriptors of molecules (without using 3-D information) has been defined with data taken from experiments regarding activity/inactivity of organic molecules as templates for the synthesis of BEA, BEC and ISV zeolites. Software based on linear discrimination analysis was able to select topological descriptors that maximize activity for each zeolite depending on the value of the discriminant function (DF) defined in each case. Using a large database, 29 molecules with DF values in the predicted range of activity were preselected and their zeolite-SDA dispersion energy, that quantifies the energetic stability of the zeolite-SDA system, was calculated for all these 29 SDAs in BEA, BEC, ISV at full loading using zeoTsda software (based in Monte-Carlo and Lattice Energy Minimization). The results allow the discovery of new templates that are predicted to be selective for either BEA, BEC or ISV. Having similar micropores makes it difficult to find selective templates for either BEA, BEC or ISV, but in spite of this difficulty, 13 SDAs have been suggested as potentially selective SDAs. Future synthesis experiments will allow the methods presented here to be refined interactively.

Models based on molecular topology, with the definition of several hundred related properties for molecules, have been applied to organic structure directing agents for the first time. The technique has been proved to work appropriately. Improving the quality and enlarging the number of molecules of the training set may allow the selection of candidate templates in a more accurate way.

As far as we know, none of the 29 SDAs proposed has been reported as SDA for the synthesis of any zeolite. This is in part because none of the databases of chemical compounds that we have 
used for screening contains SDAs for zeolites. This was a desired outcome of this strategy. A large majority of SDAs for zeolite are cations, whilst the databases we have so far used contain only neutral molecules. More simple compounds than those reported here have been used as templates. For instance, some of the piperazine derivatives employed as SDAs for zeolites are 1,4-dimethyl-piperazine, 1,4-diethyl-piperazine, and 1-methyl-piperazine, which are more simple than our piperazine derivatives (SDA10-13, SDA16, SDA26-27). Current work is ongoing to try the synthesis of these zeolites (BEA, BEC, ISV) using some of the 29 SDAs suggested.

\title{
ASSOCIATED CONTENT
}

Supporting Information

Mechanistic interpretation of indices. Information about training set and internal validation of QSAR models. Selection of SDAs for the synthesis of BEA, BEC, ISV by applying the topological models. Comparison of zeoTsda performance and coordinates (CIF files) of zeo-SDA systems of related previous work. Chem-Draw pictures of 29 preselected candidate SDAs for the synthesis of BEA, BEC, ISV. Full details of Monte Carlo+ Lattice Energy Minimization algorithm and comparison of current results with those in reference 27. Figures of optimized geometries of SDA-zeolite systems described in Table 2, and their corresponding coordinates (CIF files).

\author{
AUTHOR INFORMATION \\ Corresponding author \\ E-mail: gsastre@itq.upv.es (G.S.) (Phone: +34-963-879-445) \\ ORCID
}

Maria Gálvez-Llompart 0000-0002-0718-4143

Jorge Galvez 0000-0003-0928-8437

Fernando Rey 0000-0003-3227-5669

German Sastre: 0000-0003-0496-6331

Notes

The authors declare no competing financial interest.

\section{Acknowledgements}

We thank MICINN of Spain for funding through projects RTI2018-101784-B-I00, RTI2018101033-B-I00, and SEV-2016-0683. M.G.L. thanks GVA for a postdoctoral contract APOSTD/2019/055. The authors thank ASIC-UPV and SGAI-CSIC for the use of their computational facilities. 


\section{References}

(1) Jiang, J.; Yu, J.; Corma, A. Extra-large-pore Zeolites: Bridging the Gap between Micro and Mesoporous Structures. Angew. Chem., Int. Ed. Engl. 2010, 49, 3120-3145.

(2) Li, J.; Corma, A.; Yu, J. Synthesis of New Zeolite Structures. Chem. Soc. Rev. 2015, 44, 7112-7127.

(3) Jiang, J.; Xu, Y.; Cheng, P.; Sun, Q.; Yu, J.; Corma, A.; Xu, R. Investigation of Extralarge Pore Zeolite Synthesis by a High-throughput Approach. Chem. Mater 2011, 23, 47094715 .

(4) Zeolites and catalysis: synthesis, reactions and applications; Cejka, J.; Corma, A.; Zones, S, Eds.; John Wiley \& Sons: Weinheim, Germany, 2010.

(5) Rimer, J. D. Rational Design of Zeolite Catalysts. Nat. Catal. 2018, 1, 488-489.

(6) Lew, C.; Davis, T.; Elomari, S. In Synthesis of new molecular sieves using novel structure-directing agents; Mintova, S, Eds.; Synthesis Commission of the International Zeolite Association: Caen, France, 2016, Chapter 2, 29-35.

(7) Bell, R. G.; Lewis, D. W.; Voigt, P.; Freeman, C. M.; Thomas, J. M.; Catlow, C. R. A.; Computer Modelling of Sorbates and Templates in Microporous Materials; Stud. Surf. Sci. Catal. 1994, 84, 2075.

(8) Boyett, R. E.; Stevens, A. P.; Ford, M. G.; Cox, P. A.; A Quantitative Shape Analysis of Organic Templates Employed in Zeolite Synthesis; Zeolites 1996, 17, 508.

(9) Lobo, R. F.; Zones, S. I.; Davis, M. E.; Structure-direction in Zeolite Synthesis; J. Inclus. Phenom. Mol. 1995, 21, 47.

(10) Jackowski, A.; Zones, S. I.; Hwang, S.-J.; Burton, A. W.; Diquaternary Ammonium Compounds in Zeolite Synthesis: Cyclic and Polycyclic N-Heterocycles Connected by Methylene Chains ; J. Am. Chem. Soc. 2009, 131, 1092-1100.

(11) Archer, R. H. ; Zones, S. I.; Davis, M. E.; Imidazolium Structure Directing Agents in Zeolite Synthesis: Exploring Guest/host Relationships in the Synthesis of SSZ-70; Microporous Mesoporous Mater. 2010, 130, 255-265.

(12) Schmidt, J. E.; Deem, M. W.; Davis, M. E.; Synthesis of a Specified, Silica Molecular Sieve by Using Computationally Predicted Organic Structure-Directing Agents; Angew. Chem. Int. Ed. 2014, 53, 8372-8374.

(13) Smeets, S.; McCusker, L. B.; Baerlocher, C.; Elomari, S.; Xie, D.; Zones, S. I.; Locating Organic Guests in Inorganic Host Materials from X-ray Powder Diffraction Data; J. Am. Chem. Soc. 2016, 138, 7099-7106.

(14) Lewis, D. W.; Willock, D. J.; Catlow, C. R. A.; Thomas, J. M.; Hutchings, G. J.; De Novo Design of Structure-directing Agents for the Synthesis of Microporous Solids; Nature 1996, 382, 604 .

(15) Casci, J. L.; Cox, P. A.; Henney, R. P. G.; Maberly, S.; Shannon, M. D.; Template Design for High Silica Zeotypes: a Case Study of Zeolite NES Synthesis Using a Designed Template; Stud. Surf. Sci. Catal. 2004, 154, 110.

(16) Turrina, A.; Garcia, R.; Cox, P. A.; Casci, J. L.; Wright, P. A.; Retrosynthetic CoTemplating Method for the Preparation of Silicoaluminophosphate Molecular Sieves; Chem. Mater. 2016, 28, 4998-5012.

(17) Schmidt, J. E.; Deem, M. W.; Lew, C.; Davis, T. M.; Computationally-Guided Synthesis of the 8-Ring Zeolite AEI; Top. Catal. 2015, 58, 410-415.

(18) Schmidt, J. E.; Deem, M. W.; Davis, M. E.; Synthesis of a Specified, Silica Molecular Sieve by Using Computationally Predicted Organic Structure-Directing Agents; Angew. Chem. Int. Ed. 2014, 53, $8372-8374$.

(19) Daeyaert, F.; Deem, M. W.; Design of Organic Structure Directing Agents for Polymorph A Zeolite Beta; J. Mater. Chem. A 2019, 7, 9854-9866. 
(20) Pophale, R.; Daeyaert, F.; Deem, M. W.; Computational Prediction of Chemically Synthesizable Organic Structure Directing Agents for Zeolites; J. Mater. Chem. A 2013, 1, 6750-6760.

(21) Daeyaert, F.; Deem, M. W.; A Pareto Algorithm for Efficient De Novo Design of Multifunctional Molecules, Mol. Inf. 2017, 36, 1600044.

(22) Sabater, M. J.; Sastre, G.; "A Computational Study on the Templating Ability of the Trispyrrolidinium Cation in the Synthesis of ZSM-18 Zeolite"; Chem. Mater. 2001, 13, 4520.

(23) Sastre, G.; Leiva, S.; Sabater, M. J.; Gimenez, I.; Rey, F.; Valencia, S.; Corma, A.; "Computational and Experimental Approach to the Role of Structure Directing Agents in the Synthesis of Zeolites: the Case of Cyclohexyl Alkyl Pyrrolidinium Salts in the Synthesis of Beta, EU-1, ZSM-11 and ZSM-12 Zeolites"; J. Phys. Chem. B 2003, 107, 5432.

(24) Sastre, G.; "A Computational Chemistry Insight into the Role of Structure Directing Agents in the Synthesis of Zeolites". Phys. Chem. Chem. Phys. 2007, 9, 1052-1058.

(25) Gálvez-Llompart, M.; Cantín, A.; Rey, F.; Sastre, G. Computational Screening of Structure Directing Agents for the Synthesis of Zeolites. A Simplified Model. Z. Kristallogr. Cryst. Mater. 2019, 234, 451-460.

(26) Moliner, M.; Rey, F.; Corma, A. Towards the Rational Design of Efficient Organic Structure-directing Agents for Zeolite Synthesis. Angew. Chem., Int. Ed. Engl. 2013, 52, 13880-13889.

(27) Daeyaert, F.; Ye, F.; Deem, M. W. Machine-learning Approach to the Design of OSDAs for Zeolite Beta. Proc. Natl. Acad. Sci. U. S. A. 2019, 116, 3413-3418.

(28) Lewis D.W. In Computer Modelling of Microporous Materials; Catlow, C.; van Santen, R.; Smit, B, Eds.; Elsevier, Ltd. 2004.

(29) Carr, D. A.; Lach-hab, M.; Yang, S.; Vaisman, I. I.; Blaisten-Barojas, E. Machine Learning Approach for Structure-based Zeolite Classification. Micropor. Mesopor. Mat. 2009, 117, 339-349.

(30) Schwalbe-Koda, D.; Jensen, Z.; Olivetti, E.; Gómez-Bombarelli, R. Graph Similarity Drives Zeolite Diffusionless Transformations and Intergrowth. Nat. Mat. 2019, 18, 1177-1181.

(31) Todeschini, R.; Consonni, V. Handbook of molecular descriptors; John Wiley \& Sons: 2008.

(32) Torrens, F.; Castellano, G. Fractal Dimension of Active-site Models of Zeolite Catalysts. J. Nanomater. 2006, 2006, Article ID 17052.

(33) Leflaive, P.; Pirngruber, G.; Faraj, A.; Martin, P.; Baron, G.; Denayer, J. Statistical Analysis and Partial Least Square Regression as New Tools for Modelling and Understanding the Adsorption Properties of Zeolites. Micropor. Mesopor. Mat. 2010, 132, 246-257.

(34) Maier, S. M.; Jentys, A.; Lercher, J. A. Steaming of Zeolite BEA and its Effect on Acidity: a Comparative NMR and IR Spectroscopic Study. J. Phys. Chem. C 2011, 115, 80058013.

(35) Sastre, G.; Cantin, A.; Diaz-Cabañas, M. J.; Corma, A. Searching Organic Structure Directing Agents for the Synthesis of Specific Zeolitic Structures: An Experimentally Tested Computational Study. Chem. Mater. 2005, 17, 545-552.

(36) Zones, S. I.; Burton, A. W.; Lee, G. S.; Olmstead, M. M. A Study of Piperidinium Structure-directing Agents in the Synthesis of Silica Molecular Sieves under Fluoride-based Conditions. J. Am. Chem. Soc. 2007, 129, 9066-9079.

(37) Moliner, M.; Corma, A. Advances in the Synthesis of Titanosilicates: from the Medium Pore TS-1 Zeolite to Highly-accessible Ordered Materials. Micropor. Mesopor. Mat. 2014, $189,31-40$.

(38) Corma, A.; Navarro, M. T.; Rey, F.; Valencia, S. ITQ-16, a New Zeolite Family of the Beta Group with Different Proportions of Polymorphs A, B and C. Chem. Commun. 2001, 1720-1721.

(39) Liu, X.; Kasian, N.; Tuel, A. New Insights into the Degermanation Process of ITQ-17 Zeolites. Micropor. Mesopor. Mat. 2014, 190, 171-180.

(40) Botella, P.; Corma, A.; Navarro, M.; Rey, F.; Sastre, G. On the Shape Selective Acylation of 2-methoxynaphthalene over Polymorph C of Beta (ITQ-17). J. Catal. 2003, 217, 406-416. 
(41) Paillaud, J.; Lorgouilloux, Y.; Harbuzaru, B.; Caullet, P.; Patarin, J.; Bats, N. Structure orienting role of germanium in zeolite synthesis; Stud. Surf. Sci. Catal. 2007, 170, 389-396.

(42) Moliner, M.; Serna, P.; Cantín, Á.; Sastre, G.; Díaz-Cabañas, M. J.; Corma, A. Synthesis of the Ti- silicate Form of BEC Polymorph of $\beta$-zeolite Assisted by Molecular Modeling. $J$. Phys. Chem. C 2008, 112, 19547-19554.

(43) Burton, A. W.; Zones, S. I.; Elomari, S. The Chemistry of Phase Selectivity in the Synthesis of High-silica Zeolites. Curr. Opin. Colloid Interface Sci. 2005, 10, 211-219.

(44) Corma, A. Towards a Rationalization of Zeolite and Zeolitic Materials Synthesis; Stud. Surf. Sci. Catal. 2004, 154, 25-40.

(45) Villaescusa, L.; Díaz, I.; Barrett, P.; Nair, S.; Lloris-Cormano, J.; Martínez-Mañez, R.; Tsapatsis, M.; Liu, Z.; Terasaki, O.; Camblor, M. Pure Silica Large Pore Zeolite ITQ-7: Synthetic Strategies, Structure-directing Effects, and Control and Nature of Structural Disorder. Chem. Mat. 2007, 19, 1601-1612.

(46) Cantin, A.; Corma, A.; Diaz-Cabanas, M.; Rey, F.; Sastre, G. A New Synthesis Route of the Tridirectional 12-ring Channel Zeolite ITQ-7; Stud. Surf. Sci. Catal. 2004, 154, 481-488.

(47) Song, J.; Marler, B.; Gies, H. Synthesis of ITQ-7 with a New Template Molecule and its Crystal Structure Analysis in the As-synthesized Form. Comptes Rendus Chim. 2005, 8, 341352.

(48) Sastre, G.; Fornes, V.; Corma, A. On the Preferential Location of Al and Proton Siting in Zeolites: a Computational and Infrared Study. J. Phys. Chem. B. 2002, 106, 701-708.

(49) Blay, V.; Gullon-Soleto, J.; Galvez-Llompart, M.; Galvez, J.; Garcia-Domenech, R. Biodegradability Prediction of Fragrant Molecules by Molecular Topology. ACS Sustain. Chem. Eng. 2016, 4, 4224-4231.

(50) Dragon for Windows, version 5.4; software for molecular descriptor calculations; Talete Srl.: Milano, Italy, 2006.

(51) Lachenbruch, P. A.; Goldstein, M. Discriminant Analysis. Biometrics 1979, 35, 69-85.

(52) Statistica, version 9; Data Analysis Software System; StatSoft, I.: Tulsa, USA, 2009.

(53) Klecka, W.R.; Iversen,G.R.; Klecka, W.R. Discriminant Analysis. (Sage University Paper series on Quantitatives Applications in the Social Science, No. 07-019). Sage: Beverly Hills, CA, USA, 1980.

(54) Matthews, B. W. Comparison of the Predicted and Observed Secondary Structure of T4 Phage Lysozyme. Biochim. Biophys. Acta, Protein Struct. 1975, 405, 442-451.

(55) Roy, K.; Kar, S.; Das, R. N. In Statistical Methods in QSAR/QSPR; A Primer on QSAR/QSPR Modeling; Springer: 2015; pp 37-59.

(56) Fawcett, T. ROC graphs: Notes and Practical Considerations for Researchers. Mach. Learn. 2004, 31, 1-38.

(57) Demler, O. V.; Pencina, M. J.; D'Agostino Sr, R. B. Equivalence of Improvement in Area under ROC Curve and Linear Discriminant Analysis Coefficient under Assumption of Normality. Stat. Med. 2011, 30, 1410-1418.

(58) Veerasamy, R.; Rajak, H.; Jain, A.; Sivadasan, S.; Varghese, C. P.; Agrawal, R. K. Validation of QSAR Models-strategies and Importance. Inter. J. Drug Des. Discov. 2011, 3, 511-519.

(59) Gramatica, P. Principles of QSAR Models Validation: Internal and External. QSAR Comb. Sci. 2007, 26, 694-701.

(60) Gálvez, J.; García-Domenech, R.; de Gregorio Alapont, C.; de Julián-Ortiz, J.; Popa, L. Pharmacological Distribution Diagrams: a Tool for De Novo Drug Design. J. Mol. Graph. 1996, 14, 272-276.

(61) Sterling, T.; Irwin, J. J. ZINC 15. Ligand Discovery for Everyone. J. Chem. Inf. Model. 2015, 55, 2324-2337.

(62) Specs database. Screening compounds collection. https://www.specs.net/index.php?page=login (accessed May 25, 2020).

(63) Asinex database ASINEX. ASINEX Platinum Collection. http://www.asinex.com (accessed May 25, 2020).

(64) eMolecules database. https://www.emolecules.com/ (accessed May 25, 2020). 
(65) Catlow, C.; Bell, R.; Gale, J.; Lewis, D. Modelling of Structure and Reactivity in Zeolites; Stud. Surf. Sci. Catal. 1995, 97, 87-100.

(66) Corma, A.; Díaz, U.; García, T.; Sastre, G.; Velty, A. Multifunctional Hybrid OrganicInorganic Catalytic Materials with a Hierarchical System of Well-defined Micro- and Mesopores. J. Am. Chem. Soc. 2010, 132, 15011-15021.

(67) Gale, J. D.; Rohl, A. L. The General Utility Lattice Program (GULP). Mol. Simul. 2003, 29, 291-341. 